\title{
Acoustic softening and stress superposition in ultrasonic vibration assisted uniaxial tension of copper foil: experiments and modeling
}

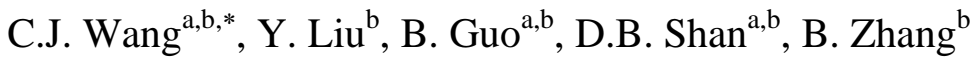 \\ ${ }^{a}$ National Key Laboratory for Precision Hot Processing of Metals, Harbin 150001, China \\ ${ }^{\mathrm{b}}$ School of Materials Science and Engineering, Harbin Institute of Technology, Harbin 150001, China
}

\begin{abstract}
Ultrasonic vibration is widely utilized in manufacturing processes mainly because acoustic field could significantly affect the metal plasticity leading to stress reduction. However, viewpoints on the influence mechanism have not reached a consensus yet. In this paper, an ultrasonic vibration assisted uniaxial tension experiment with copper foils is carried out using a specially-developed device. The results show that the extent of stress reduction increases with the increase of the vibration amplitude. Acoustic softening and stress superposition are both considered in a developed model to describe the stress reduction due to ultrasonic excitation during metal forming process. Considering ultrasonic vibration provides the energy for dislocation sliding, acoustic softening is analyzed based on crystal plasticity theory considering ultrasonic intensity. Stress superposition, mostly induced by the additional periodic strain, is included by taking account of its proportional relationship with vibration amplitude. The calculation results from the numerical model show a good agreement with those from the experiment. These findings provide an instructive understanding of mechanism of stress reduction in ultrasonic vibration assisted metal deformation and is especially helpful for pro-actively designing ultrasonic vibration assisted metal forming processes.
\end{abstract}

Keywords: Ultrasonic vibration; Stress reduction; Hybrid model; Acoustic softening; Stress superposition

\section{Introduction}

Since the application of ultrasonic vibration into the tension test of single crystal zinc by Blaha and Langenecker in 1955 [1], vibration assisted forming has been studied extensively due to its advantages of reducing flow stress [2], lowering friction between die-specimen interface [3], and improving surface finish

\footnotetext{
${ }^{*}$ Corresponding author. Tel.: +86 0451 86418640; fax: +86 045186418640.

E-mail address: cjwang1978@ hit.edu.cn (C.J. Wang).
} 
[4], especially in the field of micro forming [5-7]. By applying ultrasonic vibration (or high-frequency vibration) during the deformation processes for the metallic materials, significant progress has been achieved in contrast with traditional processes [2]. In recent years, vibration assisted technology had fast developed and been well applied into micro extrusion [8,9], wire drawing [10, 11], welding [12], and other related fields [13-15]. Nevertheless, the underlying mechanism of the effect on metal plasticity induced by ultrasonic vibration is still unclear and controversial; stress superposition, acoustic softening, and friction decrease are the three generally accepted explanations [16].

The stress superposition mechanism could be depicted by the correlation between stress-strain curve and time-strain curve $[17,18]$, based on a rate-independent elastic-plastic material in an idealized condition. According to this explanation, the material will experience both elastic deformation and plastic deformation within one period of oscillation. After this continuous oscillation the average stress can be reduced accordingly. Compared with stress superposition, acoustic softening explains the mechanism from the viewpoint of dislocation motion, which is reckoned as a way of changing the material property. According to this, ultrasonic vibration energizes the dislocation that improves the migration and multiplication [18]. For hard materials or work-hardened materials, energy that delivered to internal structure will be able to reduce dislocation density, and lead to a stress reduction at a macro scale level during deformation $[19,20]$. Besides, the thermal effect induced by acoustic softening is also included in the mechanism study of ultrasonic vibration [2, 21-23]. When the vibration is applied on metallic material, the internal particles will be activated and there will be more relative motion generated, both of which can raise the temperature to some extent and render a thermal softening. As a result, the overall dynamic deformation resistance is correspondingly reduced. The acoustic softening is generally regarded as the main reason for flow stress reduction in the case of high amplitude and high energy density [16]. According to these explanations, crystal plasticity theory based constitutive models have been developed to predict the deformation induced by ultrasonic vibration. Siddiq $[24,25]$ developed a material model by modifying the viscoplastic powerlaw expression of plastic slip system proposed by Hutchinson [26], in which the ultrasonic softening effect, $U_{\text {soff }}$, and the thermal softening effect, $T_{\text {soft }}$, are considered and integrated to the strain hardening term. Parameters in the model are identified by using inverse modeling approach. This model is capable of capturing not only crystal orientation effect but also softening effect. Yao [27] proposed an acoustic softening model and an acoustic residual hardening model based on thermal activation theory and 
dislocation evolution theory, respectively. Upsetting tests assisted by high-frequency vibration are conducted and the results show a good agreement with the model prediction. In addition, an S-shaped increase of the stress related to the vibration duration was observed after vibration is removed. For the acoustic softening effect, however, investigations also illustrate that no significant temperature rise is observed during vibration assisted experiments [17, 21, 28]. As reported in quite a few literatures, the flow stress decrease due to acoustic softening is proportional to the vibration amplitude (acoustic energy density) $[2,29]$. While whether the stress decrease is independent upon the frequency or the temperature is still disputable $[24,30]$. Therefore, fundamental research on vibration assisted forming need to go deeper and wider. Although mechanical behavior experiments, especially for vibration assisted compression processes, are widely investigated, few reports focus on tension tests of metallic foil.

In order to describe the underlying mechanism of the stress reduction under ultrasonic field, a specialized device for ultrasonic vibration assisted uniaxial tension was developed. The vibrator including ultrasonic transducer and ultrasonic horn was designed systematically. Tension tests with copper foil were carried out to investigate the mechanism of stress reduction induced by ultrasonic vibration. According to the studies having been reported for now, the stress reduction due to ultrasonic vibration was generally explained by either acoustic softening (relating to dislocation motion) or stress superposition. In this paper, both acoustic softening and stress superposition were taken into account as an outcome of stress reduction; an analytical model was developed to describe the plastic deformation behavior of metal materials under the excitation of ultrasonic vibration. The effect of amplitude of ultrasonic vibration on material property is also discussed in details.

\section{Experimental setup and materials}

\subsection{Vibration assisted tension device}

The ultrasonic vibration assisted uniaxial tension device consists of a traditional tension testing machine and an ultrasonic vibrator driven by ultrasonic generator. As one of the main module, the vibrator adopts PZT (piezoelectric ceramic transducer) with sandwich structure, which can effectively produce a longitudinal vibration. The plan sketch of the vibrator is shown in Fig. 1. Considering the amplitude limitation of the ultrasonic transducer, an ultrasonic horn is needed. The optimum length of the vibrator is a half of ultrasonic wavelength. According to the size of the mould in the future work, the vibrator length is 
designed to $70 \sim 80 \mathrm{~mm}$, while the ultrasonic wavelength is limited in 140 160 mm. The vibration propagation formula is as following:

$v=\lambda f$

where $v$ is the velocity of ultrasonic wave in metallic media, $\lambda$ is ultrasonic wavelength, and $f$ is ultrasonic frequency. Since the velocity of ultrasonic wave in 45 steel is $5200 \mathrm{~m} / \mathrm{s}, f$ should be $32.5 \sim 37.1 \mathrm{kHz}$ calculated by Eq. (1). In this paper, the working frequency of the vibrator is finally chosen to be $35 \mathrm{kHz}$. In order to meet the strength requirement in tension test and to improve working efficiency, type PZT-8 is chosen as the ultrasonic transducer.

As can be seen in Fig. 1, the flange surface is appointed as the "zero" displacement section which divides the vibrator into a quarter-wavelength ultrasonic transducer and a quarter-wavelength ultrasonic horn. Based on transmission line theory, frequency equation of the ultrasonic transducer is expressed as:

$\frac{z_{3}}{z_{2}} \tan k_{2} L_{2} \tan k_{3} L_{3}+\frac{z_{3}}{z_{1}} \tan k_{1} L_{1} \tan k_{3} L_{3}+\frac{z_{2}}{z_{1}} \tan k_{1} L_{1} \tan k_{2} L_{2}=1$

where $z$ is wave impedance defined as $z=\rho v S, \rho$ is density, $v$ is wave velocity, $S$ is section area, $k$ is number of waves defined as $k=\omega / v, \omega$ is circular frequency, $L$ is length of each section. Frequency equation of the ultrasonic horn is expressed as:

$\tan k_{4} L_{4} \tan k_{5} L_{5}=\frac{z_{4}}{z_{5}}$

In term of Eq. (2) and Eq. (3), dimensions of every section including length and diameter were calculated as shown in Table 1.

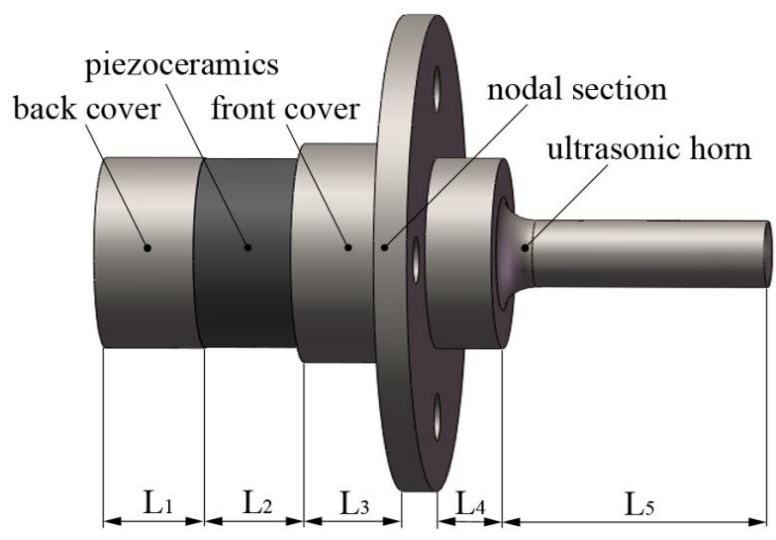

Fig. 1. Structure of the vibrator. 
Table 1

Dimensions of the vibrator.

\begin{tabular}{llllll}
\hline Sections & $\mathrm{L}_{1}$ & $\mathrm{~L}_{2}$ & $\mathrm{~L}_{3}$ & $\mathrm{~L}_{4}$ & $\mathrm{~L}_{5}$ \\
\hline Length $(\mathrm{mm})$ & 10.5 & 10.5 & 8 & 5.5 & 26.5 \\
\hline Diameter $(\mathrm{mm})$ & 20 & 20 & 22 & 19 & 5.8 \\
\hline
\end{tabular}

The natural frequency of the vibrator is analyzed using commercial software Abaqus. Different vibration modes are extracted and the results show that the 9th order vibration mode with a frequency of $35.38 \mathrm{kHz}$ agrees well with the theoretical design. In order to meet the requirement of being a clamp in the tension test, the vibrator needs to be processed into a groove structure at the horn tip. Considering the effect of this groove structure on natural frequency of the vibrator, different dimensions of the grooves have been analyzed by Abaqus. After simulation and optimization, the groove width and groove depth are finally designed as $0.2 \mathrm{~mm}$ and $10 \mathrm{~mm}$ respectively, as shown in Fig. 2. The vibration mode of the grooved vibrator is numerically analyzed again and the results show that the 11 th order vibration mode with a longitudinal frequency of $35.82 \mathrm{kHz}$ and a frequency offset below $1 \mathrm{kHz}$ meet the initial design demand, as shown in Fig. 3.

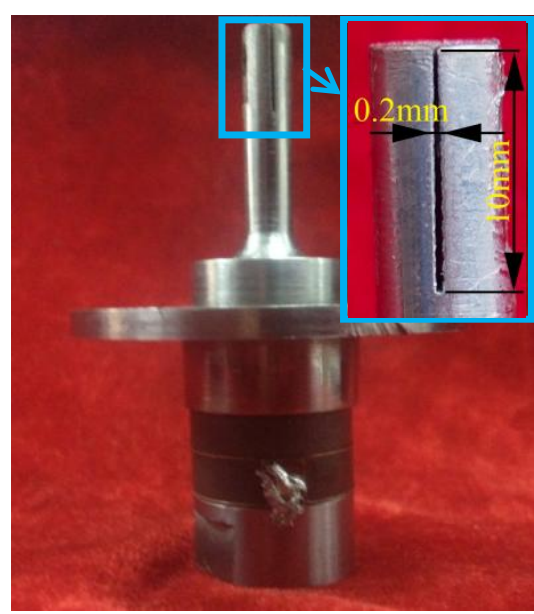

Fig. 2. The grooved vibrator.

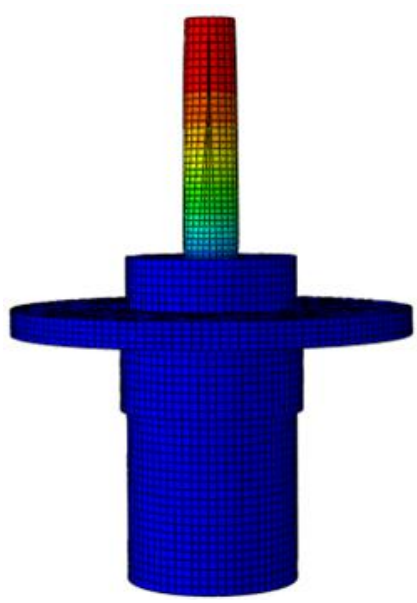

Fig. 3. The 11th order vibration mode.

To verify the practical performance of the vibrator, laser vibrometer Polytec OFV-5000 is used to measure the longitudinal vibration frequency and the amplitude of vibrator, and they are $35.21 \mathrm{kHz}$ and $0.735 \mu \mathrm{m}$ respectively, which fulfill the requirement for this test. The ultrasonic vibration assisted uniaxial tension device is shown in Fig. 4. 


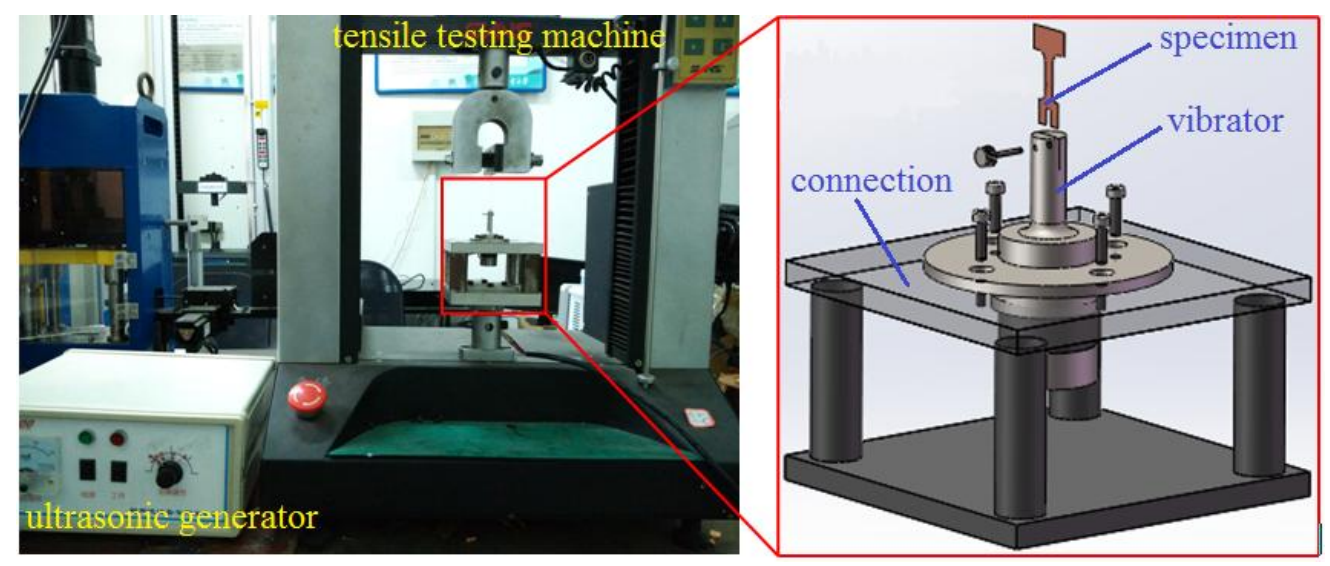

Fig. 4. Ultrasonic vibration assisted uniaxial tension device.

\subsection{Experimental material}

Copper is widely used in different fields of manufacturing due to its good formability and corrosion resistance. As the development of micro-forming technology, copper is playing an important role in the pressure processing of complex shape micro parts and antimagnetic materials, and is also regarded as high performance metallic material in the field of micro-forming. Thereby, copper foil $\mathrm{T} 2$ is chosen as the material to be investigated. The thickness of the copper foil sample is $100 \mu \mathrm{m}$. Chemical component of copper foil $\mathrm{T} 2$ is shown in Table 2.

Table 2

Chemical components of copper foil T2 (\%).

\begin{tabular}{lllllll}
\hline $\mathrm{Cu}$ & $\mathrm{Bi}$ & $\mathrm{Sb}$ & $\mathrm{Pb}$ & $\mathrm{As}$ & $\mathrm{S}$ & $\mathrm{O}$ \\
\hline$\geq 99.90$ & $\leq 0.002$ & $\leq 0.002$ & $\leq 0.005$ & $\leq 0.002$ & $\leq 0.005$ & $\leq 0.006$ \\
\hline
\end{tabular}

In order to research the effect of ultrasonic vibration on copper foils of different grain sizes, the initial rolled copper foil is annealed with different temperatures. After annealing processing, the copper foil is polished mechanically and electrolytically in sequence. Metallographs of different grain sizes are obtained with metalloscope, as shown in Fig. 5. The average grain size is measured and its deviation SD is calculated based on ASTM E112. In the ultrasonic vibration assisted uniaxial tension test, in order to match the groove structure of the vibrator, tension specimen is designed with a fork-shaped end (Fig. 6). 

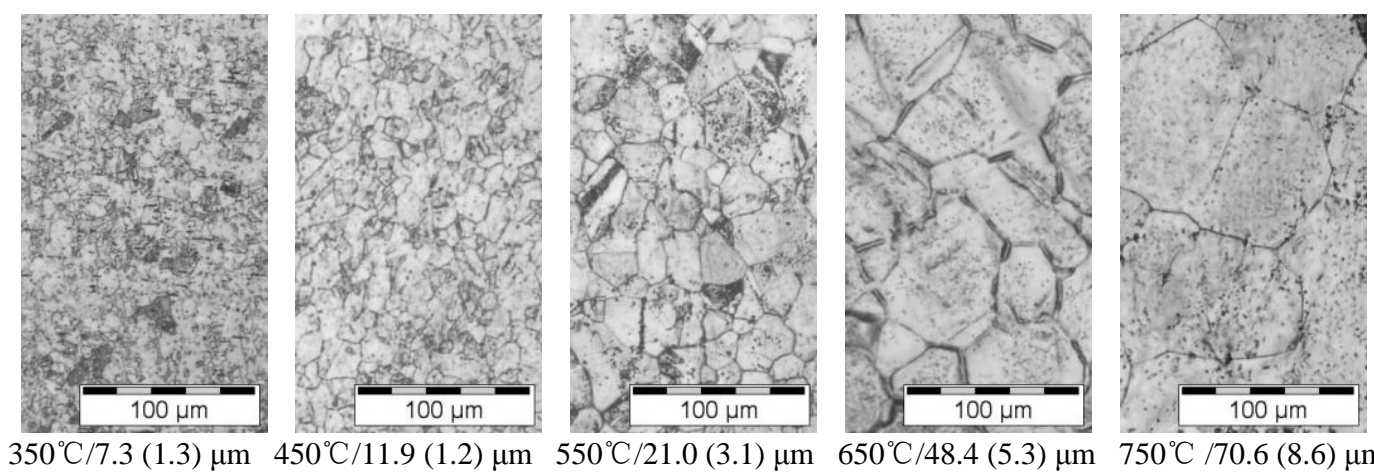

Fig. 5. Metallographs of different grain size (deviation SD) at different annealing processes.

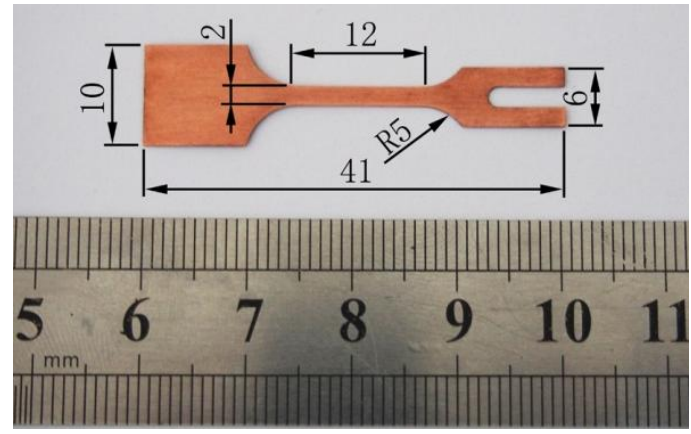

Fig. 6. Specimen with a fork-shaped end.

Plastic deformation behavior of the copper foil under ultrasonic vibration is experimentally investigated with the developed tension device. Tension tests are conducted with and without vibration at the tension speed of $2 \mathrm{~mm} / \mathrm{s}$. The effects of ultrasonic vibration on mechanical properties of different grain-sized specimens are analyzed in details.

\section{Experimental results}

Copper foil $\mathrm{T} 2$ presents special mechanical properties differing from general tension test when ultrasonic vibration is applied during uniaxial tension. As shown in Fig. 7, the stress decreases when ultrasonic vibration is applied; when the ultrasonic vibration is removed, the stress returns back to normal immediately. This phenomenon is known as "Blaha effect", which shows a stress reduction in metallic materials during deformation assisted with ultrasonic vibration. In order to investigate the influence of "Blaha effect" on different grain-sized materials, copper foil specimens with grain size of $7.3 \mu \mathrm{m}, 11.9 \mu \mathrm{m}$, $21.0 \mu \mathrm{m}, 48.4 \mu \mathrm{m}$ and $70.6 \mu \mathrm{m}$ are tested with and without ultrasonic vibration, respectively. The ultrasonic vibration is applied at the onset of the tension test until the specimens fracture. The tension conditions for 
different grain-sized specimens with or without ultrasonic vibration are kept as consistent, including the time point of applying ultrasonic vibration. Copper is regarded as a metal material without clear yield point in the tension test, so the stress corresponding to $0.2 \%$ of the plastic strain is regarded as its yield strength. According the true stress-strain curve, the yield strengths for different grain-sized specimens with and without vibration are obtained as shown in Fig. 8.

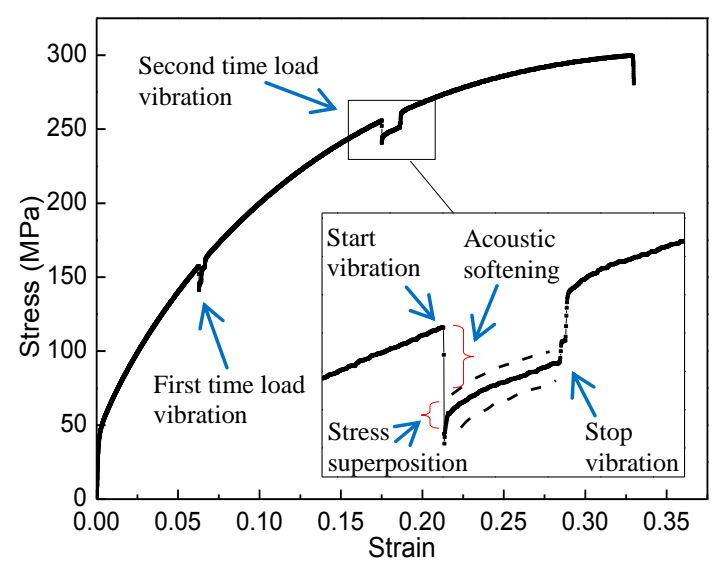

Fig. 7. Softening effect induced by ultrasonic vibration.

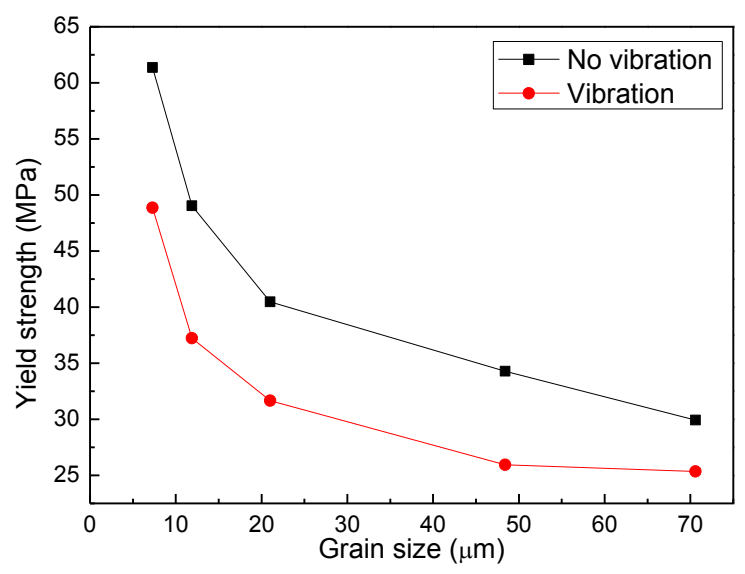

Fig. 8. Effect of vibration on yield strength.

As can be seen, the ultrasonic vibration reduces the yield strength of the copper foil, showing a stress reduction effect on metallic materials. One of the explanations that has been widely accepted is acoustic softening. When the ultrasonic vibration is applied, activity of particles inside the material will increase due to the absorption of acoustic energy which promotes the sliding and multiplication of dislocations. Intense dislocation motion further produces pile-up group at the grain boundaries and activates the adjacent dislocations. Therefore, the yield strength and the flow stress during the deformation are reduced. Meanwhile, the yield strength increases with the decrease of grain size, no matter the vibration is applied or not. This confirms the reinforcement effect of grain boundary on metal strength. 
The deformation feature of copper foil is greatly influenced by ultrasonic vibration conditions. Vibration amplitude is considered as one of the most important parameters during vibration assisted forming. Therefore, different values of amplitude are used to study their effect on the yield strength of copper foil with the grain size of $21.0 \mu \mathrm{m}$. The test result in Fig. 10 shows that the yield strength decreases with the increase of vibration amplitude. According to the dislocation mechanism, when the metal absorbs the acoustic energy and dislocation energy increases, which makes the sliding and multiplication of dislocations occur easily and further leads to a reduction of deformation resistance. As a result, the larger of the vibration amplitude is applied, the more drop of the yield strength [29, 31].

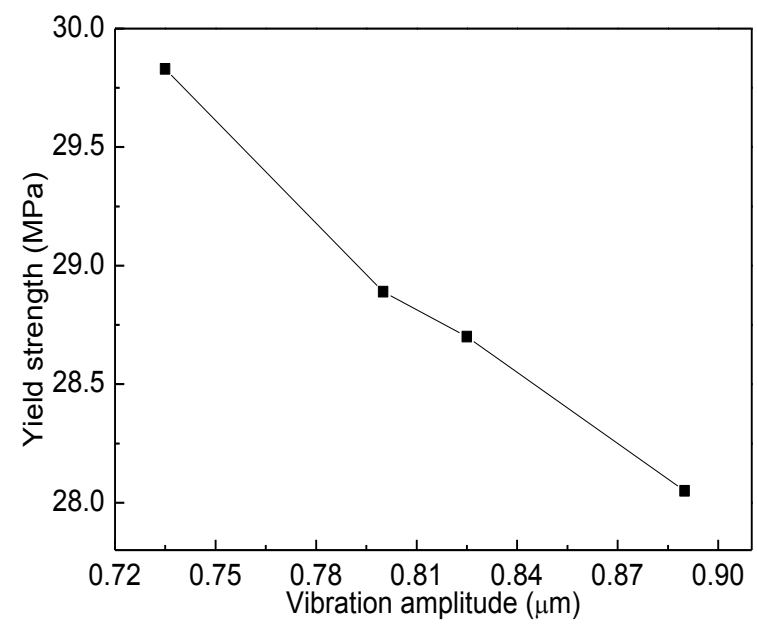

Fig. 10. Effects of vibration amplitude on yield strength.

\section{Model development}

The yield point is the beginning for plastic deformation and features with the dislocation behavior on a microscopic scale. In order to simplify the modeling process, yield stress is used for the calculation and verification of the following hybrid model.
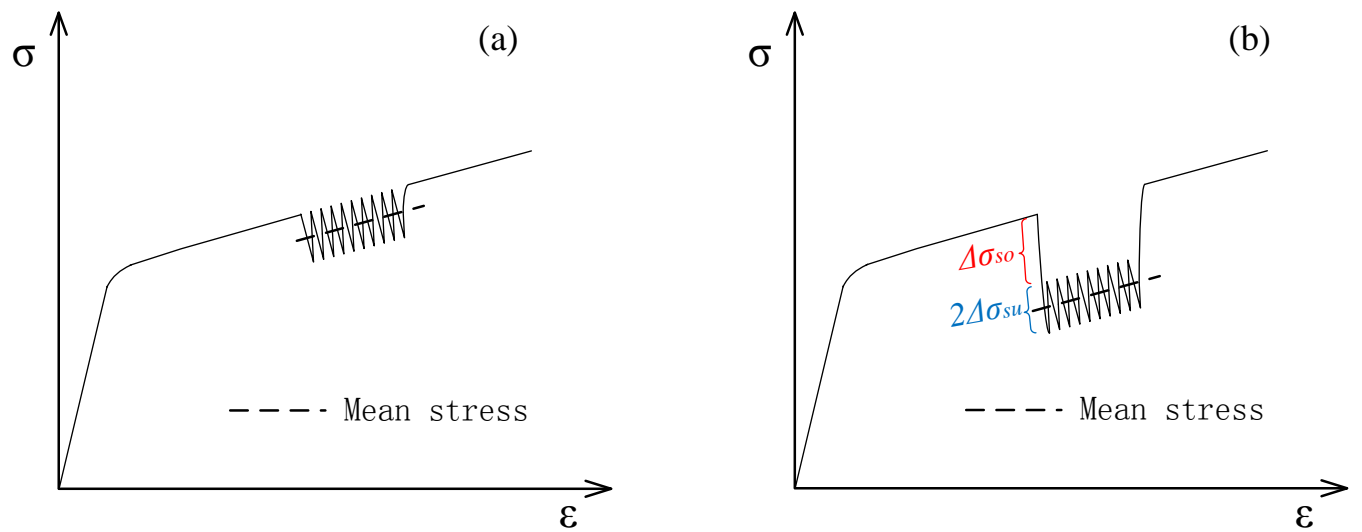

Fig. 11. Schematic diagram of stress reduction due to ultrasonic excitation: (a) Stress superposition (b) Acoustic softening and stress superposition. 
Basically, the effect of ultrasonic vibration on metal plastic deformation is explained by stress superposition, acoustic softening, and friction decrease. Since there is no contact condition in tension test, the effect of friction decrease could be neglected. The thermal effect is also ignored because the specimen is miniature and its thickness is very thin. In Fig. 11, the way of stress reduction due to ultrasonic excitation is described. If stress superposition is the only reason that causing the stress reduction, the path of the maximum oscillatory stress is supposed to follow the path of static stress and the amplitude of the oscillatory stress will only depend on the amplitude of the ultrasonic vibration, as shown in Fig. 11 (a). However, the experimental data departs from this prediction. The experimental data shows a significant reduction in the maximum, mean and minimum oscillatory stress, which makes the maximum stress no longer follow the static stress, as shown in Fig. 11 (b). Meanwhile, dynamic response of the specimen or the fixture such as resonance, which may be considered as the reason leading to the case in Fig. 11 (b), has been excluded by monitoring the whole system during ultrasonic tension test using a laser vibrometer [32]. So in this study, the stress reduction due to ultrasonic vibration is attributed to a combination effect of stress superposition and acoustic softening, just as shown in Fig. 11 (b). A model for predicting plastic deformation behavior of metal under the excitation of ultrasonic vibration is then supposed as follows:

$$
\Delta \sigma_{s}=\Delta \sigma_{s o}+\Delta \sigma_{s u}
$$

where $\Delta \sigma_{s}$ is the total reduction of yield stress, $\Delta \sigma_{s o}$ is the yield reduction due to acoustic softening, $\Delta \sigma_{s u}$ is the yield reduction due to stress superposition.

\subsection{Model development for acoustic softening}

The acoustic softening is well explained by a model based on thermal activation theory and verified in the vibration assisted compression test [27]. The model making connection between the acoustic energy density $(E)$ and the net-change in stress ratio due to acoustic softening $(\Delta \lambda)$, could be expressed as:

$$
\Delta \lambda=-\beta(E / \hat{\tau})^{m}
$$

where $\beta$ and $m$ are parameters to be determined in experiments, $\lambda$ is the stress ratio defined as $\tau / \hat{\tau}$, and therefore, $\Delta \lambda$ can also be defined as $\Delta \tau / \hat{\tau}$, where $\hat{\tau}$ is mechanical threshold of a given material and can be calculated and expressed by using the critical resolved shear stress expression as following:

$$
\hat{\tau}=\tau /\left[1-k T \ln \left(\dot{\gamma}_{0} / \dot{\gamma}_{p}\right) / \Delta F\right]
$$


where $k$ is the Boltzmann constant $(1.3807 \mathrm{E}-23 \mathrm{~J} / \mathrm{K}) ; T$ is the Kelvin temperature $(293 \mathrm{~K}) ; \dot{\gamma}_{0}$ is the preexponential factor $\left(10^{6} / \mathrm{s}\right) ; \quad \dot{\gamma}_{p}$ is the shear plastic strain rate $\left(10^{-1} / \mathrm{s}\right) ; \Delta F$ is the activation energy that can be calculated as $\Delta F=0.5 G b^{3}$, where $G$ is the shear modulus (39.2 GPa) and $b$ is the length of the Burgers vector $(0.256 \mathrm{~nm}) ; \tau$ is the critical resolved shear stress in the active slip system, which could be related to the yield stress $\sigma_{s}(42.06 \mathrm{MPa})$ via the Taylor model:

$M=\sigma_{s} / \tau$

where $M$ is the Taylor factor (3.06).Combining Eq. (6) and Eq. (7), the mechanical threshold $\hat{\tau}$ is then calculated to be 17.145 MPa. Also, with Eq. (7) the net-change in stress ratio due to acoustic softening $\Delta \lambda$ can be expressed as:

$\Delta \lambda=\Delta \tau / \hat{\tau}=\Delta \sigma_{s o} / M \hat{\tau}$

Combining Eq. (5) and Eq. (8), the stress reduction due to acoustic softening $\Delta \sigma_{s o}$ is obtained as:

$$
\Delta \sigma_{s o}=-\beta M \hat{\tau}(E / \hat{\tau})^{m}
$$

This model has correlated the yield stress reduction due to acoustic softening with the acoustic energy density. The parameters in Eq. (9) are determined based on the experimental data from the uniaxial tension of copper foil assisted with ultrasonic vibration. The acoustic energy density $E$ is related to the vibration amplitude and angular frequency, and there will be energy loss during the transmission from vibration horn tip to the experimental material. Generally, the transmission coefficient can be calculated as:

$$
\alpha_{t}=4 \rho_{C u} c_{C u} \rho_{F e} c_{F e} /\left(\rho_{C u} c_{C u}+\rho_{F e} c_{F e}\right)^{2}
$$

where $\rho$ is the material density and $c$ is the wave speed for copper and ferrum. The wave speed $c$ can be calculated as $c=\sqrt{G / \rho}$, where $G$ is the shear modulus. The transmission coefficient $\alpha_{t}$ is calculated to be 0.9793. Therefore, the acoustic energy density transmitted to the experimental material is calculated as:

$$
E=\xi_{C u}^{2} \omega^{2} \rho_{C u}=\xi_{F e}^{2} \omega^{2} \rho_{F e} \alpha_{t}
$$

where $\xi$ and $\omega$ are vibration amplitude and angular frequency, respectively. Generally, the bigger the vibration amplitude, the more energy will be produced, and the more obvious the softening effect is. 
According to the tension test, the acoustic energy density $E$ and the stress ratio $\Delta \lambda$ in Eq. (8) are calculated based on the vibration parameters and test results, as shown in Table 3.

Table 3

Calculation results of acoustic energy density and stress ratio.

\begin{tabular}{l|lllll}
\hline \multirow{3}{*}{ Vibration parameters } & Amplitude $\xi(\mu \mathrm{m})$ & 0.74 & 0.8 & 0.83 & 0.89 \\
\cline { 2 - 6 } & Vibration frequency $f(\mathrm{kHz})$ & 35.21 & 34.34 & 33.36 & 32.50 \\
\cline { 2 - 6 } & Angular frequency $\omega\left(10^{5} \mathrm{rad} / \mathrm{s}\right)$ & 2.21 & 2.16 & 2.10 & 2.04 \\
\hline \multirow{5}{*}{ Experimental data } & $\begin{array}{l}\text { Yield stress reduction due to stress } \\
\text { superposition } \Delta \sigma_{\text {su }}(\mathrm{MPa})\end{array}$ & 2.02 & 2.21 & 2.34 & 2.47 \\
\cline { 2 - 6 } & $\begin{array}{l}\text { Yield stress reduction due to acoustic } \\
\text { softening } \Delta \sigma_{\text {so }}(\mathrm{MPa})\end{array}$ & 10.21 & 10.96 & 11.02 & \multirow{2}{*}{11.54} \\
\hline \multirow{2}{*}{ Calculation parameters } & Acoustic energy density $E\left(\mathrm{~J} / \mathrm{m}^{3}\right)$ & 205.76 & 229.55 & 233.55 & 253.41 \\
\cline { 2 - 6 } & Stress ratio $\Delta \lambda$ & 0.1946 & 0.2089 & 0.21 & 0.22 \\
\hline
\end{tabular}

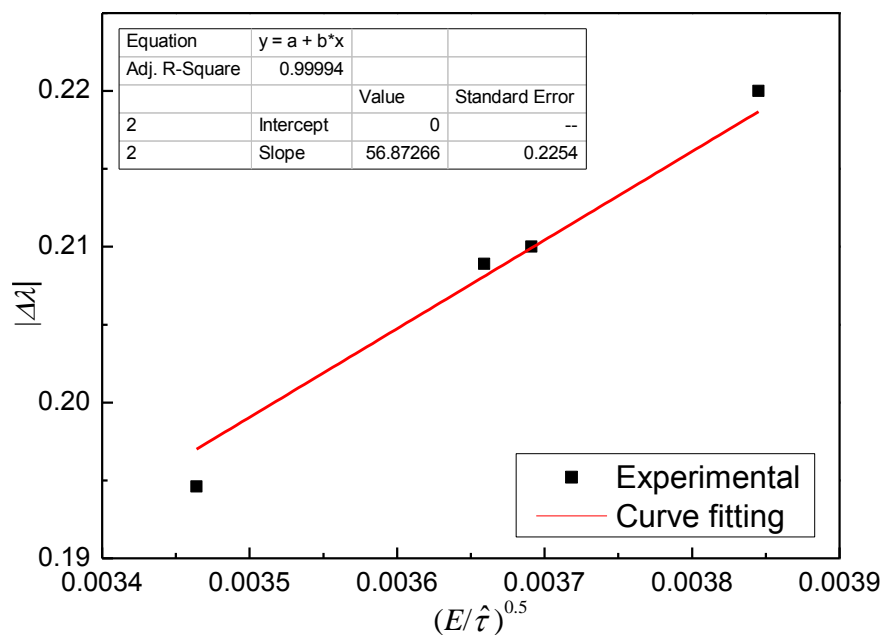

Fig. 12. Dependence of stress ratio on acoustic energy density.

The stress ratio $\Delta \lambda$ increases along with the increase of acoustic energy density $E$, as can be seen in Table 1. In order to determine the parameter $\beta$ in Eq. (4) or Eq. (8), the relationship of $\Delta \lambda$ and $(E / \hat{\tau})^{0.5}$ is depicted in Fig. 12. The parameter $m$ is considered to be 0.5 in terms of the study in which the stress reduction is observed to be proportional to the square root of the acoustic energy density [25]. The fitting curve in Fig. 12 shows a proportionality between $|\Delta \lambda|$ and $(E / \hat{\tau})^{0.5}$. As a result, $\beta$ is obtained to be 56.87 . Therefore, the model in Eq. (8) can be expressed as:

$$
\Delta \sigma_{s o}=-56.87 M \hat{\tau}(E / \hat{\tau})^{0.5}
$$




\subsection{Model development for stress superposition}

The stress superposition was initially proposed to explain the stress reduction during metal forming assisted by ultrasonic vibration. As the research deeply develops, stress superposition has been proved insufficient in explaining the decrease of deformation resistance, though it still plays its part during the process. From Table 3, the influence of stress superposition on stress reduction is much less than that of acoustic softening (about 1/5). So the stress superposition is considered to be the secondary factor causing the stress reduction. According to the stress-strain curves under different vibration amplitudes, the yield stress reduction due to stress superposition $\Delta \sigma_{s u}$ is obtained. The variation of yield stress reduction with vibration amplitude due to stress superposition is shown in Fig. 13.

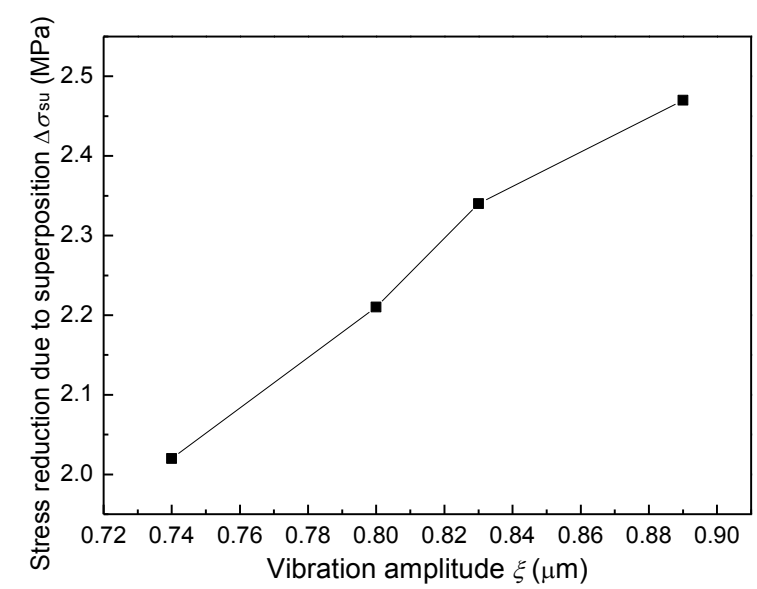

Fig. 13. The relation of vibration amplitude and yield stress reduction due to stress superposition.

Assuming the elastic-plastic material is rate-independent characterized by perfect elastic unloading in stress-strain curve, the specimen will be deformed elastically first and followed by plastic deformation during one period of oscillation [17]. During the tension assisted with ultrasonic vibration, the oscillation displacement of the ultrasonic horn tip that transmitted to the tension specimen will lead to additional periodic strain in the deformation area of the specimen, i.e., the vibration amplitude is roughly equivalent to the strain amplitude in the specimen. According to the experimental data in Fig. 13, the relation of stress reduction due to superposition and vibration amplitude is then considered follow a form of power law. For different metals, the vibration amplitudes that transmitted to the materials will be different, so $\alpha_{t}$ is introduced in the model. Thereby, the yield stress reduction due to stress superposition $\Delta \sigma_{s o}$ is supposed to be modeled as:

$$
\Delta \sigma_{s u}=-K\left(\alpha_{t} \xi\right)^{n}
$$


where the negative sign indicates a stress decrease, $\alpha_{t}$ is the transmission coefficient that has been calculated above, $n$ is the hardening exponent which is assumed to be 1 according to the approximate proportional relationship in Fig. 13, $K$ is the material strength coefficient and is considered as the slope of the curve in Fig. 13, which is obtained to be 2.774 by curve fitting. Consequently, the total reduction of yield stress induced by both acoustic softening and stress superposition is expressed as:

$$
\Delta \sigma_{s}=-56.87 M \hat{\tau}(E / \hat{\tau})^{0.5}-2.72 \xi
$$

The parameters including $\beta, \hat{\tau}, K$ and $\alpha_{t}$ may differ with different materials. According to the test, the theoretical calculation based on the model in Eq. (14) is compared with the experimental data, shown in Fig. 14. As can be seen, the results is nearly consistent, which demonstrates that the analytical model that has been proposed based on the ultrasonic assisted tension is able to provide a general description for the plastic deformation behavior of metal under the excitation of ultrasonic vibration.

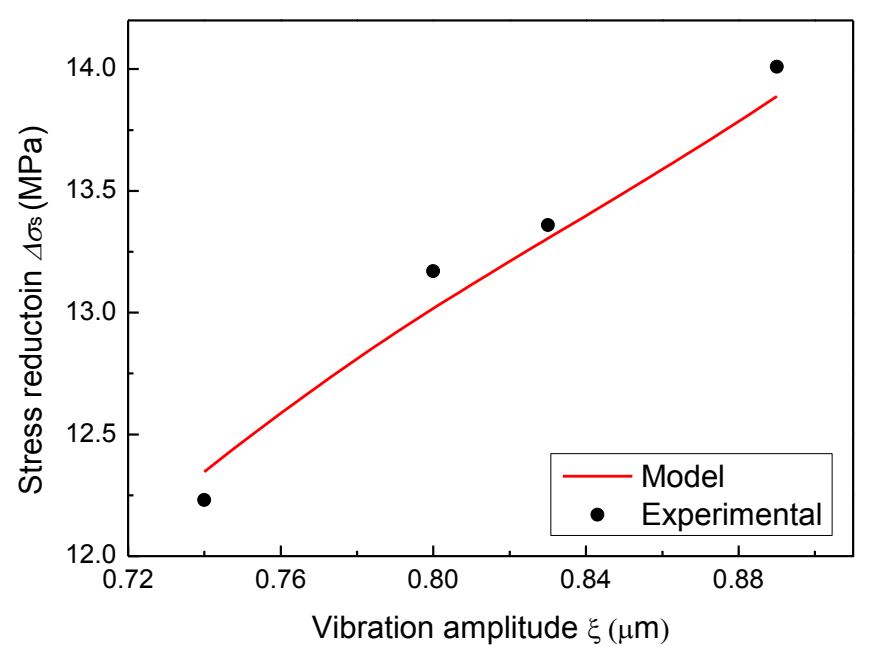

Fig. 14. The comparison of model-based calculation and experimental result.

\section{Conclusions}

An ultrasonic vibration assisted uniaxial tension device was designed, aiming at the research of stress reduction induced by ultrasonic field. The structure and dimension of ultrasonic transducer and ultrasonic horn were systematically calculated and fabricated according to the calculation. By numerical analysis and experimental measurement, the frequency of the vibrator is $35.21 \mathrm{kHz}$. The vibration amplitude, which can reflect the acoustic energy density, is within a range from $0.74 \mu \mathrm{m}$ to $0.89 \mu \mathrm{m}$ controlled by adjusting input current. Significant reduction in stress is observed when ultrasonic vibration is applied. According to the test results, acoustic energy density and stress reduction at different vibration amplitudes are calculated. In 
order to understand the underlying mechanism for the stress reduction due to ultrasonic field, an analytical model is developed considering both acoustic softening and stress superposition. Based on crystal plasticity theories, a relation is introduced between acoustic energy density and the dislocation-sliding-related softening, which describes the acoustic softening in the viewpoint of energy absorption. Considering the periodic strain, an equation is developed for calculating the stress superposition depending on the vibration amplitude. In terms of the developed model, the stress reduction of copper foil under ultrasonic field can be accurately predicted. The developed model has provided an instructive understanding for the mechanism of acoustic softening and stress superposition in ultrasonic vibration assisted metal forming.

\section{Acknowledgements}

The authors greatly appreciate the financial support from the National Science Foundation of China (No. 51375113); and the Fundamental Research Funds for the Central Universities (No. HIT. BRETIII. 201404). Authors also wish to thank Dr. Quanren Zeng for his help in writing correction.

\section{References}

[1] F. Blaha, B. Langenecker, Elongation of zinc monocrystals under ultrasonic action, Die Naturwissenschaften. 42 (1955) 556.

[2] B. Langenecker, Effects of ultrasound on deformation characteristics of metals, IEEE Trans. Son Ultrason. 13 (1966) 1-8.

[3] G.R. Dawson, C.E. Winsper, D.H. Sansome, Application of high-frequency and low-frequency oscillations to plastic deformation of metals: Part 2 - A complete appraisal of the development and potential, Met. Form. 37 (1970) 254-261.

[4] M. Malaki, H.T. Ding, A review of ultrasonic peening treatment, Mater. Des. 87 (2015) 1072-1086.

[5] C.J. Wang, B. Guo, D.B. Shan, X.M. Bai, Effects of interfacial contact stats on tribological behavior in micro sheet forming, Int. J. Mech. Sci. 101-102 (2015) 81-88.

[6] J. Xu, B. Guo, C.J. Wang, D.B. Shan, Blanking clearance and grain size effects on micro deformation behavior and fracture in micro-blanking of brass foil, Int. J. Mach. Tool. Manu. 60 (2012) 35-42.

[7] G.C. Han, K. Li, Z. Peng, J.S. Jin, M. Sun, X.Y. Wang, A new porous block sonotrode for ultrasonic 
assisted micro forming, Int. J. Adv. Manu. Tech. (2016) 1-10.

[8] J.X. Lu, X.Y. Wu, Z.Y. Liu, X.Q, Chen, B. Xu, Z.Z. Wu, S.C. Ruan, Microstructure and mechanical properties of ultrafine-grained copper produced using intermittent ultrasonic-assisted equal-channel angular pressing, Metall. Mater. Trans. A (2016) 1-11.

[9] S. Mousavi, H. Feizi, R. Madoliat, Investigations on the effects of ultrasonic vibrations in the extrusion process, J. Mater. Process. Tech. 187 (2007) pp. 657-661.

[10] C.Q. Yang, X.B. Shan, T. Xie, Titanium wire drawing with longitudinal-torsional composite ultrasonic vibration, Int. J. Adv. Mater. Tech. 83 (2016) 645-655.

[11] C.Q. Yang, X.B. Shan, T. Xie, A new piezoelectric ceramic longitudinal-torsional composite ultrasonic vibrator for wire drawing, Ceram. Int. 41 (2015) 625-630.

[12] G.K. Padhy, C.S. Wu, S. Gao, L. Shi, Local microstructure evolution in Al6061-T6 friction stir weld nugget enhanced by ultrasonic vibration, Mater. Des. 92 (2016) 710-723.

[13] T. Takemasu, S. Yamasaki, H. Miura, T. Ozaki, Piercing process by punch striking using ultrasonic vibration-trial construction of experimental apparatus and forming property of microholes, J. Jpn. Soc. Tech. Plast. 47 (2006) 885-889.

[14] W. Zhai, Z.Y. Hong, X.L. Wen, D.L. Geng, B. Wei. Microstructural characteristics and mechanical properties of peritectic Cu-Sn alloy solidified within ultrasonic field, Mater. Des. 72 (2015) 43-50.

[15] Y. Bai, M. Yang, Investigation on mechanism of metal foil surface finishing with vibration-assisted micro-forging, J. Mater. Process. Tech. 213 (2013) 330-336.

[16] M. Shalvandi, Y. Hojjat, A. Abdullah, H. Asadi, Influence of ultrasonic stress relief on stainless steel 316 specimens: A comparison with thermal stress relief, Mater. Des. 46 (2013) 713-723.

[17] B. Schinke, T. Malmberg, Dynamic tensile tests with superimposed ultrasonic oscillations for stainless steel type 321 at room temperature, Nucl. Eng. Des. 100 (1987) 281-296.

[18] Z.H. Yao, G.Y. Kim, L. Faidley, Q.Z. Zou, D.Q. Mei, Z.C. Chen, Micro pin extrusion of metallic materials assisted by ultrasonic vibration, Proceedings of the ASME 2010 International Manufacturing Science and Engineering Conference, 1-5.

[19] I. Lum, H. Huang, B.H. Chang, M. Mayer, D. Du, Y. Zhou, Effects of superimposed ultrasound on deformation of gold, J. Appl. Phys. 024905 (2009) 1-5. 
[20] K.W. Siu, A.H.W. Ngan, Understanding acoustoplasticity through dislocation dynamics simulations, Philos. Mag. 91 (2011) 4367-4387.

[21] Z.H. Yao, G.Y. Kim, L. Faidley, Q.Z. Zou, D.Q. Mei, Z.C. Chen, Effects of superimposed highfrequency vibration on deformation of aluminum in micro/ meso-scale upsetting, J. Mater. Process. Tech. 212 (2012) 640-646.

[22] O. Izumi, K. Oyama, Y. Suzuki, Effects of superimposed ultrasonic vibration on compressive deformation of metals, Trans. Jpn. Inst. Met. 7 (1966) 162-167.

[23] G.A. Malygin, Acoustoplastic effect and the stress superimposition mechanism, Phys. Solid State, 42 (2000) 72-78.

[24] A. Siddiq, T.E. Sayed, Acoustic softening in metals during ultrasonic assisted deformation via CPFEM, Mater. Lett. 65 (2011) 356-359.

[25] A. Siddiq, T.E. Sayed. A thermomechanical crystal plasticity constitutive model for ultrasonic consolidation. Comp. Mater. Sci. 51 (2012) 241-251.

[26] J.W. Hutchinson, Bounds and self-consistent estimates for creep of polycrystalline materials, Proc. R. Soc. London Ser. A 348 (1976) 101-127.

[27] Z.H. Yao, G.Y. Kim, Z.H. Wang, L. Faidley, Q.Z. Zou, D.Q. Mei, Z.C. Chen, Acoustic softening and residual hardening in aluminum: Modeling and experiments, Int. J. Plast. 39 (2012) 75-87.

[28] K.V. Sapozhnikov, S.B. Kustov, Effect of temperature on the amplitude dependences of the acoustoplastic effect and internal friction during deformation of crystals, Philos. Mag. A 76 (1997) $1153-1168$.

[29] H. Huang, A. Pequegnat, B.H. Chang, M. Mayer, D. Du, Y. Zhou, Influence of superimposed ultrasound on deformability of Cu, J. Appl. Phys. 106 (2009) 113514.

[30] J.J. Gilman, Contraction of extended dislocations at high speeds, Mater. Sci. Eng. A, 319 (2001) 8486.

[31] J.C. Hung, C.C. Lin, Investigations on the material property changes of ultrasonic-vibration assisted aluminum alloy upsetting, Mater. Des. 45 (2013) 412-420.

[32] Y. Daud, M. Lucas, Z.H. Huang, Modeling the effects of superimposed ultrasonic vibrations on tension and compression tests of aluminium, J. Mater. Process. Tech. 186 (2007) 179-190. 

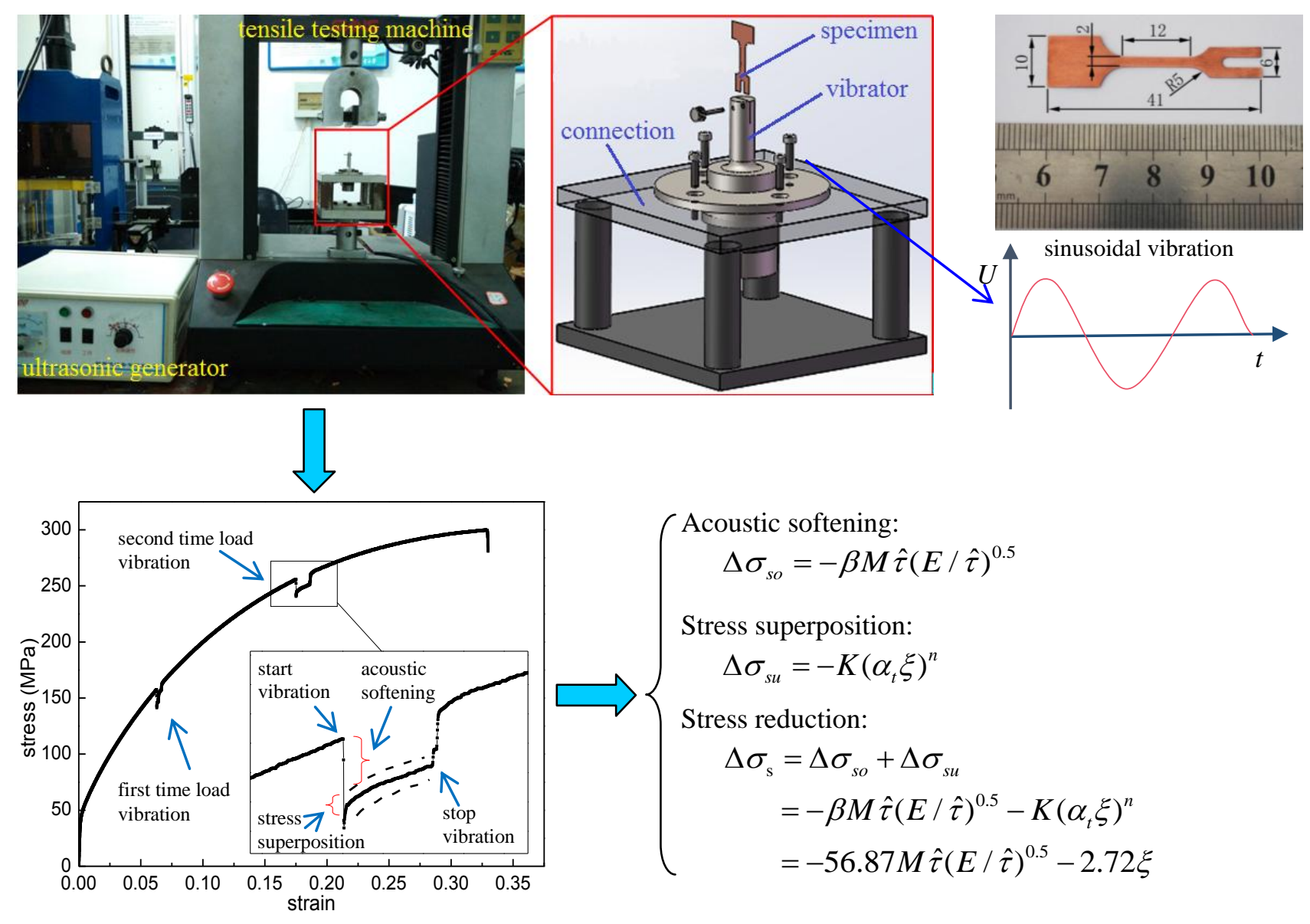